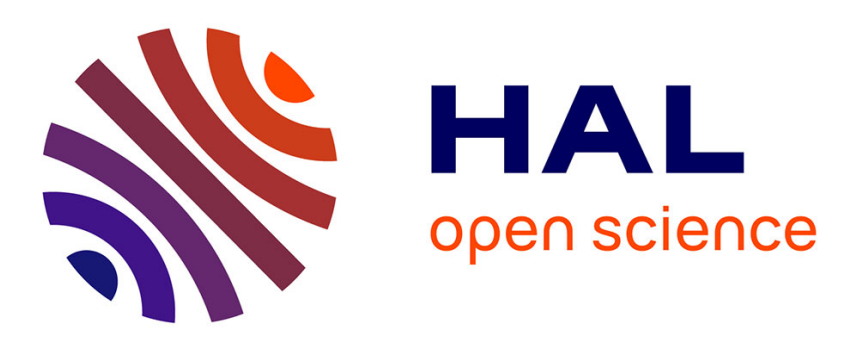

\title{
Topographically induced internal solitary waves in a pycnocline: Primary generation and topographic control
}

\author{
Yvan Dossmann, F. Auclair, A. Paci
}

\section{To cite this version:}

Yvan Dossmann, F. Auclair, A. Paci. Topographically induced internal solitary waves in a pycnocline: Primary generation and topographic control . Physics of Fluids, 2013, 25, pp.66601-1 - 66601-17. 10.1063/1.4808163 . hal-01753319

\section{HAL Id: hal-01753319 \\ https://hal.univ-lorraine.fr/hal-01753319}

Submitted on 17 May 2018

HAL is a multi-disciplinary open access archive for the deposit and dissemination of scientific research documents, whether they are published or not. The documents may come from teaching and research institutions in France or abroad, or from public or private research centers.
L'archive ouverte pluridisciplinaire HAL, est destinée au dépôt et à la diffusion de documents scientifiques de niveau recherche, publiés ou non, émanant des établissements d'enseignement et de recherche français ou étrangers, des laboratoires publics ou privés. 


\section{AIP Fluids}

Topographically induced internal solitary waves in a pycnocline: Primary generation and topographic control

Y. Dossmann, F. Auclair, and A. Paci

Citation: Phys. Fluids 25, 066601 (2013); doi: 10.1063/1.4808163

View online: http://dx.doi.org/10.1063/1.4808163

View Table of Contents: http://pof.aip.org/resource/1/PHFLE6/v25/i6

Published by the American Institute of Physics.

\section{Additional information on Phys. Fluids}

Journal Homepage: http://pof.aip.org/

Journal Information: http://pof.aip.org/about/about_the_journal

Top downloads: http://pof.aip.org/features/most_downloaded

Information for Authors: http://pof.aip.org/authors

\section{ADVERTISEMENT}

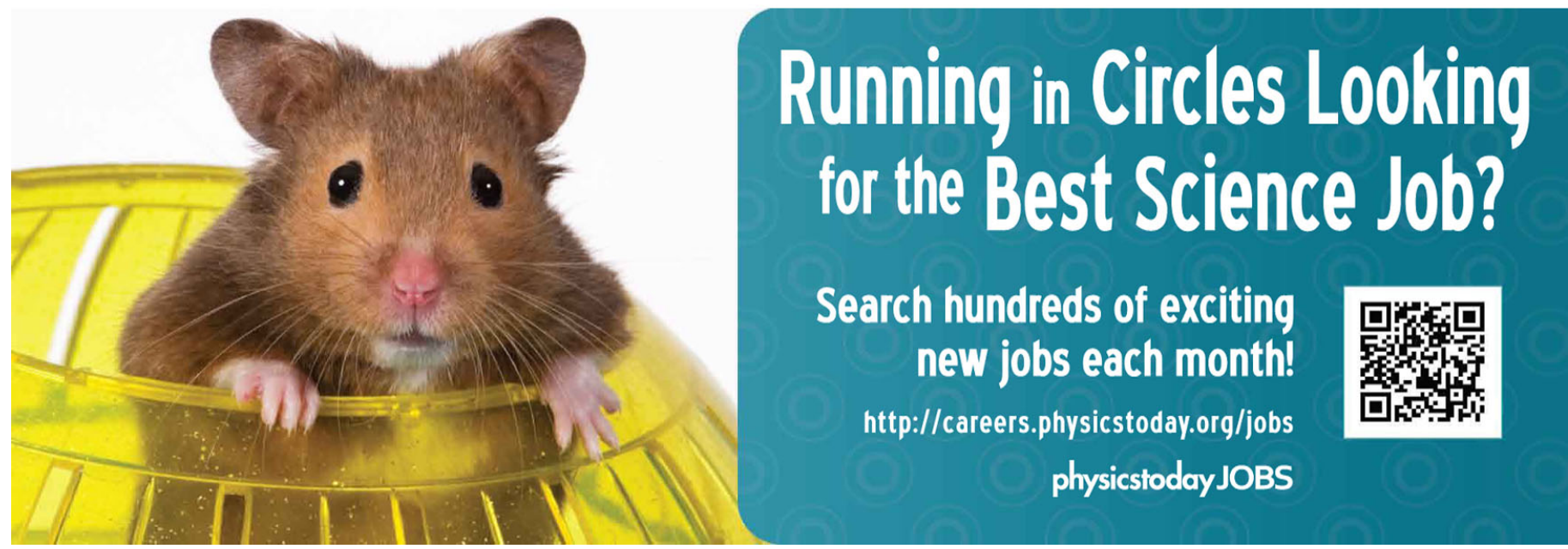




\title{
Topographically induced internal solitary waves in a pycnocline: Primary generation and topographic control
}

\author{
Y. Dossmann, ${ }^{1,2,3, \text { a) }}$ F. Auclair, ${ }^{2}$ and A. Paci ${ }^{3}$ \\ ${ }^{1}$ Research School of Earth Sciences, The Australian National University, \\ Canberra 0200, Australia \\ ${ }^{2}$ Laboratoire d'Aérologie (UMR 5560 CNRS \& UPS Toulouse III), 14 Avenue Edouard Belin, \\ 31400 Toulouse, France \\ ${ }^{3}$ CNRM-GAME/GMEI/SPEA (UMR 3589 Météo-France \& CNRS), 42 Avenue Gaspard \\ Coriolis, 31057 Toulouse Cedex 01, France
}

(Received 27 July 2012; accepted 8 May 2013; published online 3 June 2013)

\begin{abstract}
Internal solitary waves (hereafter ISWs) are stable nonlinear waves propagating in regions of strong density gradients common in geophysical flows. The purpose of the present work is to describe the generation of internal solitary waves at the interface of a two layer fluid, by the periodic oscillation of a topography. This academic configuration is inspired by oceanic observations. Direct numerical simulations, using the numerical model Symphonie-NH, are used to give insights into the physical parameters controlling the generation of these high amplitude interfacial waves in the primary generation case. The dynamics of the propagating ISWs is successfully compared with a simple Korteweg-de Vries scheme, showing that primarily generated ISWs propagate in an unimodal manner, and confirming that their stability relies on the balance between nonlinear and dispersive effects. Finally, the role of the topography in the primary generation process is quantitatively described by varying its shape. We show the existence of a topographic control of the primary generation of ISWs. A nondimensional parameter based on the ratio of the interfacial wavelength and the typical topography width is introduced to describe this spatial selection criterion. (c) 2013 AIP Publishing LLC. [http://dx.doi.org/10.1063/1.4808163]
\end{abstract}

\section{INTRODUCTION}

Internal gravity waves are involved in complex energy and momentum transfers in geophysical fluids. In regions of strong density vertical gradients that are present in the atmosphere and in the ocean, they propagate horizontally in a near interfacial manner. For large amplitudes, nonlinear effects may develop in these interfacial waves. Provided the latter are balanced by stabilizing dispersive effects, they can evolve into stably propagating internal solitary waves (ISWs). In geophysical flows, the main source of stabilizing dispersion is non-hydrostatic effects (Gerkema and Zimmerman ${ }^{1}$ ). Rotation is another source of dispersion that can play a role in the ISW stability (e.g., Grimshaw ${ }^{2}$ and Gerkema $^{3}$ ) but its effect is not tackled in this article.

The propagation of oceanic ISWs is associated with velocity shears enhancing turbulence and mixing. These dynamical processes need to be taken into account to properly represent the evolution of the ocean mixed layer (Kantha and Clayson ${ }^{4}$ ). Moreover, the strong currents and velocity shears carried by these waves are potentially hazardous to offshore operations, such as oil and gaz drilling operations (Hyder et al. ${ }^{5}$ ). The structure of ISWs also has a biological impact since important vertical velocities induced by ISWs at the pycnocline strongly affect the plankton dynamics close to the ocean surface (Lai et al. ${ }^{6}$ ). Oceanic ISWs are carrying concentrated amount of energy away from their generation zone. Consequently, they can also play a role in the redistribution of energy in the ocean.

\footnotetext{
a)yvan.dossmann@anu.edu.au
} 
The generation of ISWs in the ocean can be caused by the direct interaction between a quasihorizontal periodic flow such as the semi-diurnal tide on the one hand, and a topographic slope, such as a continental shelf or a ridge that forces an upward fluid motion on the other hand. This interaction may trigger high amplitude vertical displacements that evolve into ISWs propagating at the bottom of the ocean mixed layer (the ocean pycnocline), where the vertical density gradients are the most important. They can travel over hundreds of kilometers, before breaking or having radiated all their energy down to the abyssal ocean. The term "Primary generation" is coined to describe the generation of ISWs by this process. It has been widely observed in areas such as the Sea of Sulu where ISWs of 100-m amplitude have been measured using thermistors chains $200 \mathrm{kms}$ away from their generation location at Pearl Bank (Apel et al. ${ }^{7}$ ). At the same period, Pingree and Mardell ${ }^{8}$ measured ISWs of 50-m amplitude in the Celtic Sea, relying on a thermistor chain located $25 \mathrm{~km}$ away from the shelf break. A different type of ISWs generation process in the ocean has been convincingly described by New and Pingree. ${ }^{9}, 10$ In that case, the impingement on the pycnocline of internal waves rays emitted at the topography in the lower stratified layer leads to the generation of ISWs in the pycnocline (Gerkema, ${ }^{3}$ Akylas,${ }^{11}$ and Grisouard ${ }^{12}$ ). The latter process is not tackled in the present article that deals with the primary generation dynamics.

The dynamical properties of primarily generated ISWs depend on independent physical features, such as the flow parameters (period and amplitude), the shape of the topography, and the fluid stratification. Gerkema ${ }^{13}$ performed a theoretical study based on the Boussinesq equations for a fluid with two layers of constant densities, considering explicitly the tide-topography interaction, in which he highlighted some relevant parameters controlling the generation of high amplitude ISWs. The comparison between the numerical solution of these equations and the aforementioned in situ observations gave satisfactory results. In particular, the author noticed a strong dependence of ISW generation on the topographic shape of Pearl Bank.

Numerical simulations permitted to explore the primary generation dynamics in oceanic configurations. Using an inviscid, rigid-lid, numerical model on a rotating f-plane, Lamb ${ }^{14}$ reproduced with a good accuracy the primary generation of ISWs observed at George Bank. By running sensitivity tests, larger vertical displacements were observed for a steeper bank edge. This oceanic configuration was recently simulated with the free-surface, non-hydrostatic model used in the present article (Auclair et al. ${ }^{15}$ ). Apart from small discrepancies due to dissipative effects that are not represented in Lamb, ${ }^{14}$ the models were in close agreement. The model of Lamb ${ }^{14}$ was recently applied to simulate large amplitude ISWs generated over the ridges in Luzon Strait (Warn-Varnas et al. ${ }^{16}$ ). The forcing by three tidal components and a steady background current were represented in the model that showed a good agreement with oceanic observations. The generated ISWs matched well with a weakly nonlinear Korteweg-de Vries (KdV) analytical solution. In this configuration, the latter solution is a good approximation to describe the ISW dynamics. A KdV-type theory including rotation and considering a continuously stratified fluid was applied by New and Pingree ${ }^{17}$ to study the formation of ISWs in the bay of Biscay. The dynamical features of ISWs were well reproduced using this extended KdV analytical model.

The present study aims at describing the physical processes involved in the primary generation of ISWs using direct numerical simulations (i.e., without any turbulent closure scheme). To do so, an academic configuration at the laboratory scale inspired by oceanic cases is adopted. It permits to easily describe the role played by the topography shape, the forcing and the stratification on the primary generation process. The choice is made to work on a laboratory scale in order to compare numerical results with on-going laboratory experiments. In order to rigorously identify the simulated waves as ISWs, an analytical approach using numerically determined solutions of KdV equations is performed. We eventually aim at describing the role played by the topography shape in the primary generation process. For that purpose, different topography shapes are studied, and a simple selection criterion relying on the Fourier transform of the topography is extracted.

The article is organized as follows. In Sec. II, we present the numerical configuration adopted for this study. Section III deals with the role of nonlinear and dispersive effects on the ISW shape. In Sec. IV, the ISWs dynamics in the numerical simulations is compared with a simple and an extended $\mathrm{KdV}$ scheme. In Sec. V, the role played by the topography shape is studied. Conclusions are drawn in Sec. VI. 


\section{NUMERICAL AND PHYSICAL CONFIGURATION}

Simulations are performed using the nonhydrostatic version of the numerical model Symphonie, known as Symphonie-NH. The reader may refer to Auclair et al. ${ }^{15}$ for a substantial description of the model, for which we recall the main characteristics here.

\section{A. General features of the numerical model}

Symphonie-NH relies on the set of non-rotating, non-hydrostatic Boussinesq equations, with a linear equation of state for density in the present implementation. The discretization of the set of equations is carried out onto the Arakawa-C grid in the horizontal, and onto time-dependent, "terrain and free surface following," regularly spaced, s-levels in the vertical direction.

Free surface boundary conditions are used, while no slip conditions are used at the bottom of the flow. The temporal discretization, based on a centered leap-frog scheme, relies on a separated computation of the faster, barotropic processes associated with the free surface motions, with an external time step $\delta t_{e}$, and slower, baroclinic processes in the inner fluid, with an internal time step $\delta t_{i}=n \times \delta t_{e}$, with $n$ typically varying between 2 and 8 in the present configuration. This "mode splitting" algorithm, proposed by Blumberg and Mellor, ${ }^{18}$ permits to reduce computational costs, while describing both the fast surface and the slower inner fluid evolutions. The discretized set of Boussinesq equations used in this model are energy-conserving, as shown in Floor ${ }^{19}$ and Floor, Auclair, and Marsaleix. ${ }^{20}$

\section{B. Configuration}

Simulations are performed in a vertical plane, using a $2 \mathrm{D}$ version of the model in a vertical plane $(O x z)$, with $x=0$ at the ridge mean position, and $z=0$ at the free surface rest state position. Parallelization of the model is carried out on 16 processors, by splitting the domain of horizontal length $L$, in subdomains of equal size $L / 16$ with the MPI library.

Molecular values of the kinematic viscosity $\left(v=10^{-6} \mathrm{~m}^{2} / \mathrm{s}\right)$ and of the salinity diffusivity $\left(K_{s}\right.$ $=10^{-9} \mathrm{~m}^{2} / \mathrm{s}$ ) are used, hence they are explicitly modeled without any turbulence schemes. Periodic fluid motions are forced via oscillation of the ridge according to $x_{m}(t)=-A \cos (2 \pi t / T) \Theta(t)$, where $x_{m}(t)$ is the horizontal position of the center of the ridge, $(A, T)$ are the forcing amplitude and period, and $\Theta(t)$ is the Heaviside function. The ridge oscillates in an initially non-moving fluid, using a moving floor implemented in the model. This choice has been made in order to facilitate comparisons with on-going laboratory experiments carried out in the CNRM-GAME large stratified water flume (e.g., Knigge et al. ${ }^{21}$ ).

A set of direct numerical simulations of internal wave generation over topography is performed using this model. The configuration presented in Figure 1 is adopted.

The stratified fluid, composed of a light upper layer of depth $h_{1}=9.25 \mathrm{~cm}$ and density $\rho_{1}=1000 \mathrm{~kg} / \mathrm{m}^{3}$ and a denser bottom layer of depth $h_{2}=29.25 \mathrm{~cm}$ and density $\rho_{2}=\rho_{1}+\Delta \rho$ is initially at rest. The density stratification is controlled via salinity, at fixed temperature. The initial thickness of the continuous interface, for which the term pycnocline is used in the remainder of the article, is $d=1.5 \mathrm{~cm}$ so that the total depth equals $H=h_{1}+h_{2}+d=40 \mathrm{~cm}$. The domain length $L$ varies between $50 \mathrm{~m}$ and $200 \mathrm{~m}$ depending on the interfacial wavelength.

At $t=0$, the sinusoidal oscillation, at a period $T$, of a ridge of maximum height $h_{0}=25 \mathrm{~cm}$ and varying characteristic width $l$ and shape forces the fluid displacement. It has been shown by Gerkema and Zimmerman ${ }^{22}$ in a similar configuration that the tidal motion over a ridge is equivalent to an oscillating topography if the nonlinearity parameter $\epsilon$ introduced in Sec. III and the topography parameter $\epsilon_{b}=h_{0} / l$ are both small. In the present work, $\epsilon_{b} \leq 0.25$ and $\epsilon \leq 0.06$. The latter parameter is indeed small while the former is smaller than one but not small, indicating that discrepancies may exist between the case of a tidal forcing and the present case of an oscillating ridge.

The horizontal resolution is $d x=10 \mathrm{~cm}$ and $100 \mathrm{~s}$-levels are used, corresponding to an average resolution of $4 \mathrm{~mm}$ in the vertical direction. Although the horizontal resolution of the model is large 


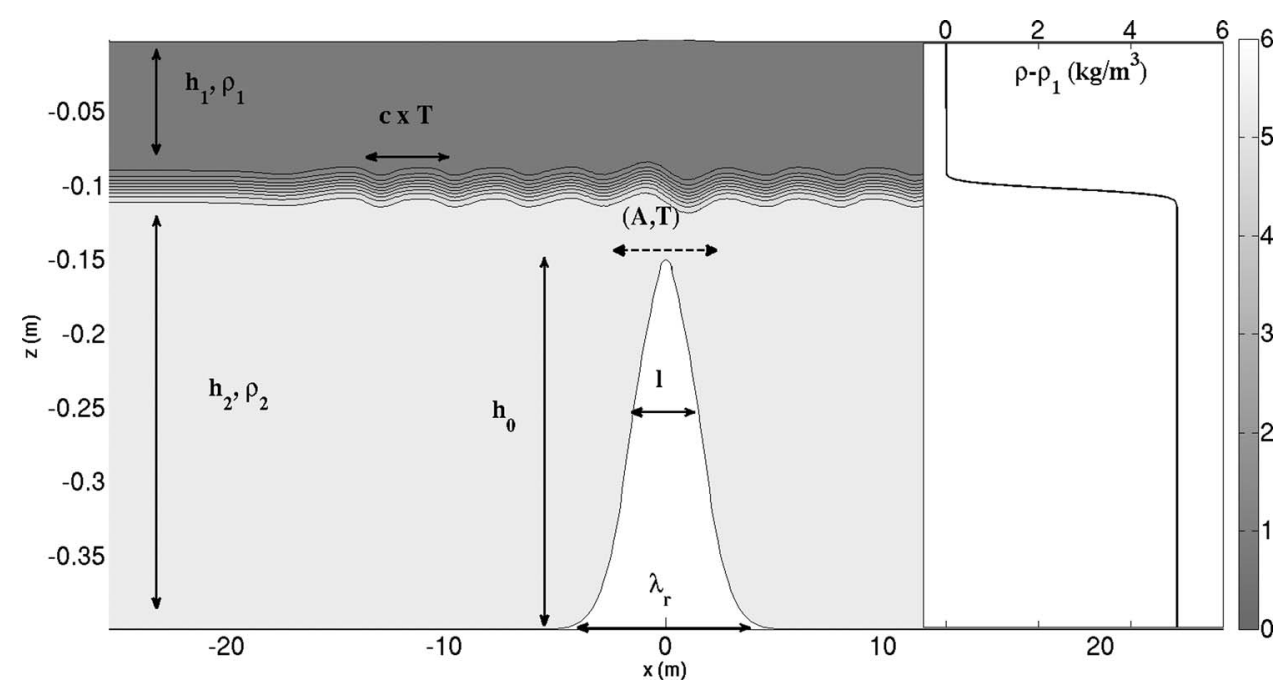

FIG. 1. Symphonie output for the density field in Sim0: 8 isopycnal lines at $t=4 T$. The horizontal scale is shrunk for visibility. The density difference between two isopycnals is $\delta \rho=0.6 \mathrm{~kg} / \mathrm{m}^{3}$. The initial density profile is shown to the right. Indicated physical parameters are defined in Table I.

compared to the mixing scales, the physical features observed have scales much larger than the numerical resolution and the relative stability of the generated waves substantially prevent an energy cascade towards scales smaller than the wavelength. Comparisons between a couple of simulations, one with $d x=1 \mathrm{~cm}$ and the other with $d x=10 \mathrm{~cm}$, confirmed this assumption. Only the mixing features in the vicinity of the ridge are better represented at $d x=1 \mathrm{~cm}$, while the observed waves are similar.

Physical parameters are varied in a range that enables to cover several regimes of interfacial wave generation. In particular, we focus on the impact of the forcing amplitude, the wavelength (controlled by the forcing period and the density jump between the two layers) and the ridge shape on the interfacial wave structure. Table I summarizes the range of parameters used in this study.

\section{NONLINEARITY/DISPERSION BALANCE}

\section{A. Nondimensional parameters}

The ISW stability relies on the balance between nonlinear effects that steepen the propagating waves and non-hydrostatic effects that disperse and flatten the waves, preventing their breaking. When these two effects are in balance, stable ISWs can propagate. To quantify these two effects, the nondimensional parameters $\epsilon$ (nonlinearity) and $\delta$ (non-hydrostaticity) introduced in the set of forced rotation-modified Boussinesq equations derived by Gerkema ${ }^{13}$ are used. These parameters are given by Eqs. (1) and (2), respectively,

$$
\begin{aligned}
\epsilon & =\frac{A}{l} \frac{h_{0}}{H}, \\
\delta & =\left(\frac{H \omega}{c^{*}}\right)^{2}, \text { with } \\
c^{*} & =\sqrt{g \frac{\Delta \rho}{\rho_{0}} \frac{h_{1} h_{2}}{H}},
\end{aligned}
$$

where the parameters $A, h_{0}, H, l, h_{1}, h_{2}, \Delta \rho, \rho_{0}$, and $\omega$ are defined in Table I. $c^{*}$ is the linear longwave speed for a wave propagating at an interface of zero thickness between two layers of depth $h_{1}$ and $h_{2}$, with a density jump $\Delta \rho$ at the interface. 
TABLE I. Numerical and physical parameters.

\begin{tabular}{|c|c|c|c|}
\hline Designation & Name & Value (Sim0) & Value (Sim0-Sim8) \\
\hline Domain length & $L(\mathrm{~m})$ & 51.2 & {$[51.2 ; 204.8]$} \\
\hline \multicolumn{4}{|l|}{ Fluid properties } \\
\hline Fluid depth & $H(\mathrm{~m})$ & 0.4 & 0.4 \\
\hline Upper layer depth & $h_{1}(\mathrm{~cm})$ & 9.25 & 9.25 \\
\hline Upper layer density & $\rho_{1}\left(\mathrm{~kg} / \mathrm{m}^{3}\right)$ & 1000 & 1000 \\
\hline Bottom layer depth & $h_{2}(\mathrm{~cm})$ & 29.25 & 29.25 \\
\hline Interface thickness & $d(\mathrm{~cm})$ & 1.5 & 1.5 \\
\hline Reference density & $\rho_{0}\left(\mathrm{~kg} / \mathrm{m}^{3}\right)$ & 1000 & 1000 \\
\hline Density jump & $\Delta \rho\left(\mathrm{kg} / \mathrm{m}^{3}\right)$ & 5 & {$[2 ; 150]$} \\
\hline Kinematic viscosity & $v\left(\mathrm{~m}^{2} / \mathrm{s}\right)$ & $10^{-6}$ & $10^{-6}$ \\
\hline Salinity diffusivity & $K_{s}\left(\mathrm{~m}^{2} / \mathrm{s}\right)$ & $10^{-9}$ & $10^{-9}$ \\
\hline \multicolumn{4}{|l|}{ Topography and forcing } \\
\hline Ridge height & $h_{0}(\mathrm{~m})$ & 0.25 & 0.25 \\
\hline Gaussian ridge e-folding width & $l(\mathrm{~m})$ & 2 & $1 ; 2$ \\
\hline Sinusoidal ridge bottom width & $\lambda_{r}(\mathrm{~m})$ & & 9 \\
\hline Forcing amplitude & $A(\mathrm{~cm})$ & 4 & {$[2 ; 20]$} \\
\hline Forcing period & $T(\mathrm{~s})$ & 60 & {$[5 ; 300]$} \\
\hline \multicolumn{4}{|l|}{ Deduced waves parameters } \\
\hline Linear longwave speed & $c^{*}(\mathrm{~m} / \mathrm{s})$ & 0.06 & {$[0.04 ; 0.3]$} \\
\hline Theoretical linear wavelength & $\lambda(\mathrm{m})$ & 3.4 & {$[2 ; 22]$} \\
\hline Nondimensional wavelength & $\lambda_{n d}$ & 0.3 & {$[0.2 ; 2.4]$} \\
\hline Nonlinearity parameter & $\epsilon$ & 0.012 & {$[0.006 ; 0.06]$} \\
\hline Dispersion parameter & $\delta$ & 0.47 & {$[0.01 ; 1.19]$} \\
\hline \multicolumn{4}{|l|}{ Numerical model parameters } \\
\hline Horizontal resolution & $d x(\mathrm{~cm})$ & 10 & 10 \\
\hline Average vertical resolution & $d z(\mathrm{~cm})$ & 0.4 & 0.4 \\
\hline External time step & $\Delta t_{e}(\mathrm{~s})$ & $3.21 \times 10^{-4}$ & $3.21 \times 10^{-4}$ \\
\hline Internal time step & $\Delta t_{i}(\mathrm{~s})$ & $1.28 \times 10^{-3}$ & $1.28 \times 10^{-3}$ \\
\hline
\end{tabular}

The parameter $\epsilon$ is proportional to the horizontal forcing amplitude $A$ times the characteristic ridge slope $h_{0} / l$ and describes the efficiency of the conversion of horizontal of the ridge into vertical motion of the interface.

The parameter $\delta$ quantifies the relative value of the fluid depth to the interfacial wavelength $2 \pi c^{*} / \omega$. For small $\delta$ (long wavelengths), non-hydrostatic effects are weak and propagating waves are non-dispersive. For higher values of $\delta$ (short wavelengths), non-hydrostatic effects disperse propagating waves packets in the pycnocline by accelerating long wavelengths.

\section{B. Nonlinear effects}

To describe the generation of topographic internal waves in the pycnocline, simulations using a steep Gaussian ridge of formula

$$
h(x)=h_{0} \exp \left(-(x / l)^{2}\right)
$$

are performed with $l=2 \mathrm{~m}$. Table II recalls the nondimensional parameters of each experiment, as well as the type of ridge used.

The back and forth oscillation of the ridge provokes the emission of two interfacial waves per period, propagating horizontally leftwards and rightwards. Figure 2 displays, from bottom to top, the evolution of the isopycnal displacement in the middle of the pycnocline between $t=1 \mathrm{~T}$ and $t=2 \mathrm{~T}$ in Sim0. The arrows to the right indicate the ridge speed, which changes direction after half a period. In the course of half a period, the pycnocline is thus displaced upwards ahead of the ridge and downwards in the lee of the ridge. Hence, in the linear case, one wavelength of the 
TABLE II. Nondimensional parameters and ridge shape for each simulation.

\begin{tabular}{lccc}
\hline \hline Simulation designation & $\epsilon$ & $\delta$ & Ridge type \\
\hline Sim0 & 0.012 & 0.476 & Gaussian \\
Sim1 & {$[0.006 \ldots 0.062]$} & 0.476 & Gaussian \\
Sim2 & 0.062 & 0.019 & Gaussian \\
Sim3 & 0.062 & 0.238 & Gaussian \\
Sim4 & 0.062 & 0.476 & Sinusoidal \\
Sim5 & 0.062 & 0.066 & Gaussian \\
Sim6 & 0.062 & 0.066 & Sinusoidal \\
Sim7 & 0.062 & {$[0.068 \ldots 1.192]$} & Gaussian \\
Sim8 & 0.062 & {$[0.015 \ldots 1.192]$} & Gaussian \\
\hline \hline
\end{tabular}

leftwards (respectively, rightwards) propagating interfacial wave is generated every period to the left (respectively, to the right), of the ridge. The measured phase speed of the wave is in good accordance with $c^{*}$, which confirms that the generated waves are indeed interfacial internal waves.

In Sim0, the generated waves present a mostly sinusoidal shape indicating that nonlinear effects are hardly present at such amplitude.

In order to study nonlinear effects in the interfacial wave, a series of 10 simulations with increasing A, called Sim1, is performed. The remaining parameters in Sim1 are the same as in Sim0. The isopycnal displacement in the middle of the pycnocline is displayed in Figure 3, after five forcing periods. For clarity, only four typical simulations of Sim1 are displayed here. Periodic structures are observed in the four cases, with a constant wavelength close to the theoretical wavelength $\lambda=c^{*}$ $\times T$ calculated in the linear longwave limit.

For $\epsilon=0.006$ (dotted line), the isopycnal displacement is close to sinusoidal, indicating mostly linear propagating interfacial waves. Nonlinear effects are visible for $\epsilon=0.018$ (dashed line) for which the wave structure consists in a sequence of broad, flat tops followed by narrow deep troughs. This structure is analogous to the cnoidal waves presented in Figure 1.4 in the dissertation of Gerkema. ${ }^{13}$

From $\epsilon=0.037$ (dashed-dotted line), a second smaller trough appears in the interfacial wave and deepens for $\epsilon=0.056$ (solid line). This sequence of troughs, directed towards the thicker layer and ranked by decreasing depth is typical of a solitary waves train (e.g., Dauxois and Peyrard ${ }^{23}$ ).

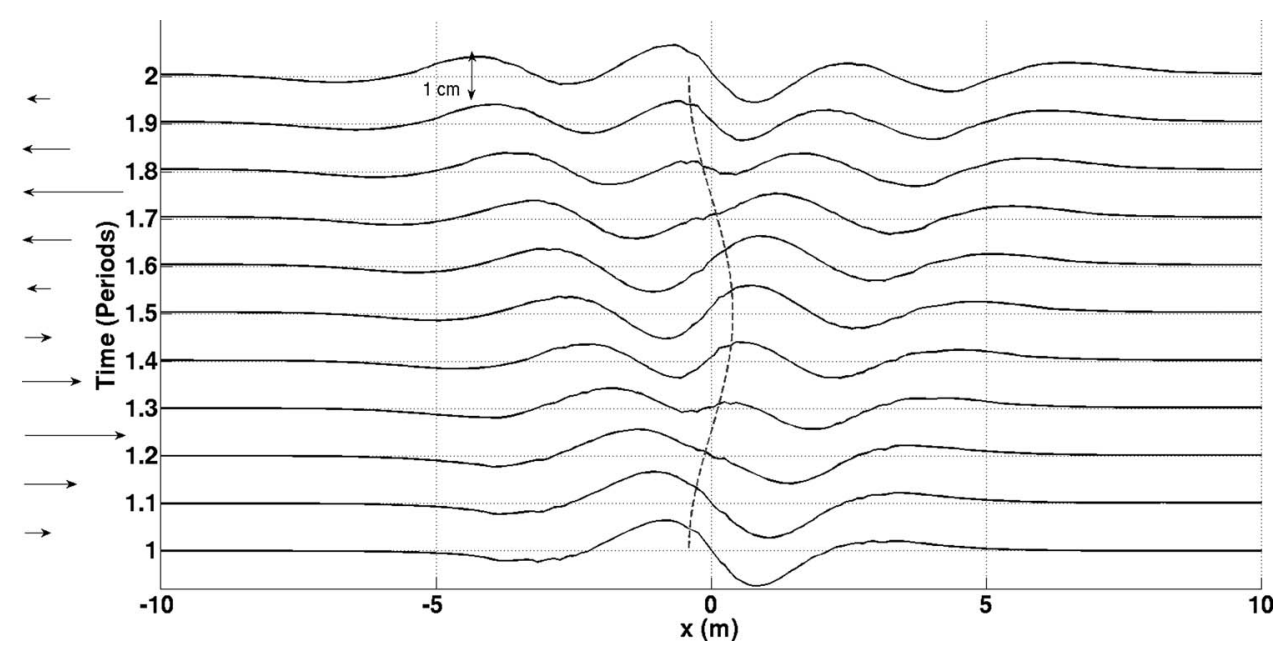

FIG. 2. Mid-pycnocline isopycnal $\left(\rho=1002.5 \mathrm{~kg} / \mathrm{m}^{3}\right)$ position at $t=1 ; 1.1 ; \ldots ; 2 T$ in Sim0, from bottom to top. Arrows on the left-hand side indicate the ridge speed. The dashed curve indicates the horizontal position of the ridge top magnified ten times. 


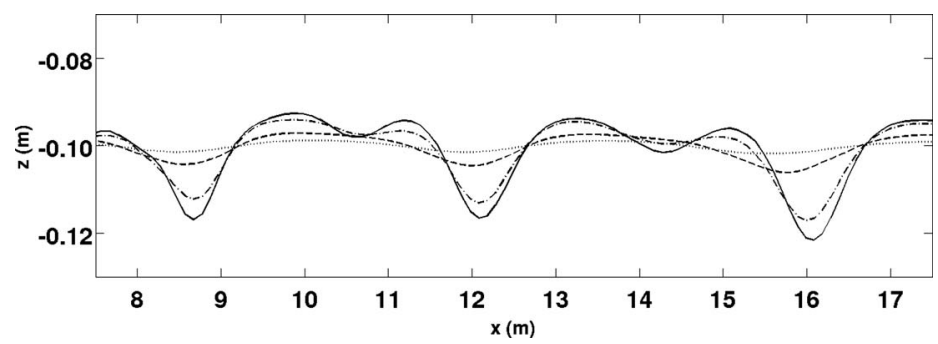

FIG. 3. Mid-pycnocline isopycnal displacement in Sim1, zoomed on two wavelengths to the right of the ridge, at $t=5 \mathrm{~T}$, for $\delta=0.47$ and $\epsilon=0.006$ (dotted line), $\epsilon=0.018$ (dashed line), $\epsilon=0.037$ (dashed-dotted line), $\epsilon=0.056$ (solid line). Nonlinearity increases as $\epsilon$ increases.

\section{Dispersive effects}

Along with showing the role of an increasing nonlinearity, Figure 3 also illustrates the competition between nonlinear and dispersive effects in the interfacial wave.

For a weak nonlinear parameter, non-hydrostatic dispersion is sufficiently strong to balance nonlinear effects in one propagating trough per period. For increasing nonlinearity, dispersive effects become too weak to oppose the nonlinear steepening of the waves. Thus, the nonlinear wave disintegrates into a sequence of depth-ranked troughs, for each of which the balance between nonlinear and dispersive effects is sustained.

The comparison between two configurations with different dispersion parameter $\delta$, and for a constant nonlinearity parameter $\epsilon$ is shown in Figure 4. The solid line shows the isopycnal displacement in the middle of the pycnocline for $\operatorname{Sim} 1(\epsilon=0.062, \delta=0.47, T=60 \mathrm{~s})$, at $t=5 \mathrm{~T}$. In $\operatorname{Sim} 2(\epsilon=0.062, \delta=0.02$, dashed line), the forcing period is increased to $T=300 \mathrm{~s}$.

The difference between the wave structures is remarkable. Due to the weaker dispersive effects in Sim2, the interfacial waves disintegrate in periodic trains of depth-ranked troughs.

An interesting comparison can be made with the expected number of ISWs $N$, expressed in terms of $\epsilon$ and $\delta$ in Eq. (5), obtained by Gerkema ${ }^{13}$ in the frame of the nondimensional inviscid forced Boussinesq equations

$$
N=\frac{1}{2}\left(\sqrt{1+\frac{6 a \epsilon(1-2 \alpha)}{\alpha(1-\alpha) \delta}}+1\right),
$$

with $a=0.2$, the typical ISW depth in Sim2 of $2 \mathrm{~cm}$ nondimensionalized by $h_{1}$, and $\alpha=h_{1} / H$.

Using this expression, one finds $N=5$ for the parameters of $\operatorname{Sim} 2$, which is close to the four troughs observed in the isopycnal displacement. The lower number of troughs in Sim2 may be explained by viscous effects that are present in numerical simulations and that tend to smooth the waves. In fact, as the scale of the isopycnal trough gets closer to the spatial resolution, smaller scale features cannot be represented. The matching between the expected number of ISWs and the number of troughs in the set of simulations is satisfactory, as the difference never exceeds 1 . Therefore, this

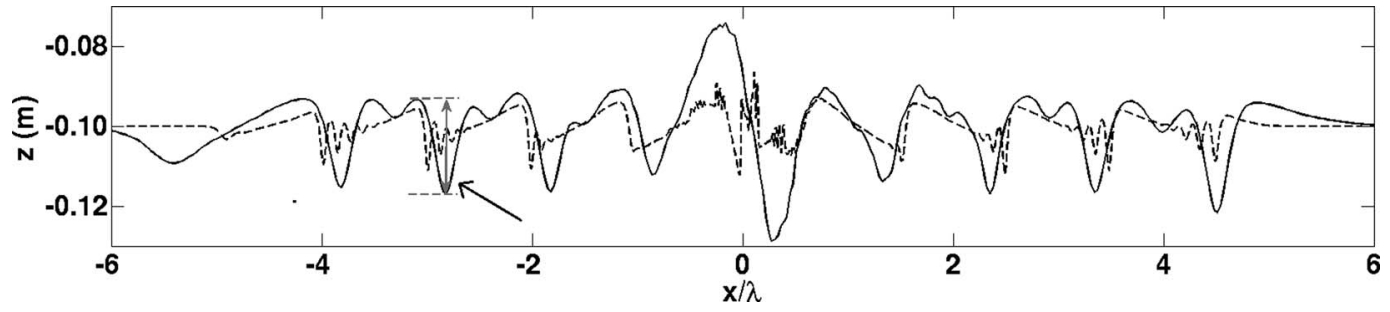

FIG. 4. Mid-pycnocline isopycnal displacement in $\operatorname{Sim1}(\epsilon=0.062, \delta=0.47$, solid line $)$ and in $\operatorname{Sim} 2(\epsilon=0.062, \delta=0.02$, dashed line), at $t=5$ T. $x$ is normalized by the linear interfacial wavelength $\lambda$ to facilitate the comparison between the two experiments. The ISW depth measured in Sec. V B is indicated in gray. 
comparison shows that the parameters $\epsilon$ and $\delta$ are relevant and sufficient to forecast the structure of a propagating nonlinear wave in the pycnocline, relying on Eq. (5).

\section{NUMERICAL MODEL VERSUS KdV SCHEME}

In order to quantitatively confirm that these nonlinear, dispersive waves do propagate as solitary waves, it is useful to compare their evolution away from the ridge, with the resolution of a theoretical equation which solutions are identified as solitary waves. In the present configuration of quasiunidimensional, nonlinearly propagating interfacial waves, a relevant equation for this test is the Korteweg-de Vries (hereafter KdV) equation.

\section{A. KdV equation}

We consider a two-layer fluid with an upper (respectively, bottom) layer of depth $h_{1}$ (respectively, $h_{2}$ ) and density $\rho_{1}$ (respectively, $\rho_{1}+\Delta \rho$ ) separated by an infinitely thin interface. The KdV equation (6) enables to describe unidirectional interfacial wave subject to weakly nonlinear and dispersive effects:

$$
\frac{\partial \eta}{\partial t}+c^{*} \frac{\partial \eta}{\partial x}+\frac{3}{2} \frac{h_{1}-h_{2}}{h_{1} h_{2}} c^{*} \eta \frac{\partial \eta}{\partial x}+\frac{1}{6} h_{1} h_{2} c^{*} \frac{\partial^{3} \eta}{\partial x^{3}}+v_{\text {num }} \frac{\partial^{2} \eta}{\partial x^{2}}=0,
$$

where $\eta(x, t)$ is the interfacial displacement. The first two terms of the $\mathrm{KdV}$ equation describe the linear propagation of the interfacial displacement, at the linear longwave speed $c^{*}$ to the right. The third nonlinear term takes the form of a supplementary advection at a speed proportional to the depth. This is consistent with the fact that solitary waves are rank-ordered by decreasing depth in a train as deeper waves are advected faster. The fourth term stabilizes the wave by dispersing the steep wave front. A parameterized diffusive term is added to sponge out the effects of numerical discretization.

A centered scheme is used to evaluate horizontal derivatives with a time-advancing scheme. Details of the discretized KdV scheme are provided in the Appendix.

\section{B. Lowest order KdV scheme}

Figure 5(a) shows the isopycnal displacement in the center of the pycnocline $\eta(x, 2 T)$ after two forcing periods for Sim3, identical to Sim1 except for the density jump which is increased to $\Delta \rho=10 \mathrm{~kg} / \mathrm{m}^{3}$.

At $t=2 T$, the wave begins to disintegrate in two steep troughs due to nonlinear effects. The interfacial displacement $\eta(x, 2 T)$ is used as an initial condition for the discretized $\mathrm{KdV}$ scheme. In Figure 5(b) is shown the isopycnal displacement $\eta(x, 3 T)$, obtained in $\mathbf{S i m 3}$, as well as the output of the KdV scheme with a numerical diffusive coefficient $v_{\text {num }}=10^{-4} \mathrm{~m}^{2} / \mathrm{s}$ (dashed line), at the same time step. The isopycnal displacements in Sim3 and in the simple KdV scheme output are very similar. The first trough of the wave, which was already formed at $t=2 T$, has the same shape in the two models. The second trough of the train that was not established at $t=2 T$, is present in the $\mathrm{KdV}$ model and also exhibits a similar shape as in Sim3. Hence, nonlinear and dispersive terms of the KdV equation reproduce with a satisfactory accuracy the transient dynamics of a high amplitude wave formation in the numerical configuration.

One can conclude from this comparison that the dynamics of the waves propagating in the pycnocline is mainly led by the balance between dispersive and nonlinear effects, away from the ridge. The interaction between the forcing flow and the topography leads, in a certain range of physical parameters, to the generation of ISWs.

\section{Extended KdV scheme}

The $\mathrm{KdV}$ equation (6) is derived under the assumption that both nonlinear and dispersive terms are weak in front of the linear wave propagation. In the case of Sim3, the depth of the isopycnal 

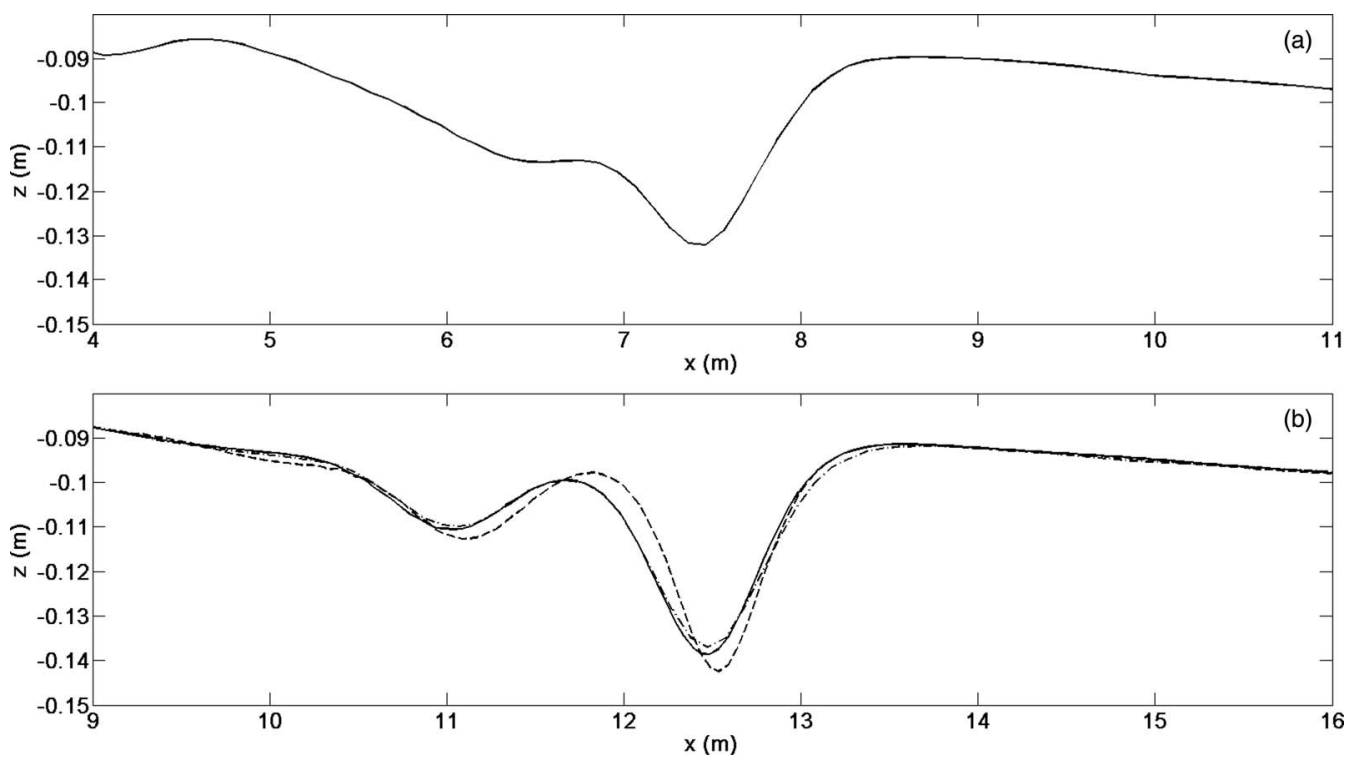

FIG. 5. (a) Mid-pycnocline isopycnal $\left(\rho=1005 \mathrm{~kg} / \mathrm{m}^{3}\right)$ in $\mathrm{Sim3}$, zoomed on one wavelength to the right of the ridge, at $t=2 T$. (b) Solid line: same as (a), but at $t=3 T$. Output of the simple KdV scheme (dashed) and of the extended KdV scheme (dashed/dotted), at $t=3 T$ (both schemes used $v_{\text {num }}=10^{-4} \mathrm{~m}^{2} / \mathrm{s}$ ). The extended $\mathrm{KdV}$ scheme permits to refine the structure of the interfacial wave, that is closer in shape to Sim3 compared to the simple KdV scheme.

trough approximately equals $h_{1} / 2$ causing potentially strong nonlinear effects. In order to represent nonlinear effects with a better accuracy, one extended version of the KdV equation including the next-order nonlinear advective term is used (Gerkema and Zimmerman ${ }^{1}$ ):

$$
\begin{aligned}
& \frac{\partial \eta}{\partial t}+c^{*} \frac{\partial \eta}{\partial x}+\frac{3}{2} \frac{h_{1}-h_{2}}{h_{1} h_{2}} c^{*} \eta \frac{\partial \eta}{\partial x}+\frac{1}{6} h_{1} h_{2} c^{*} \frac{\partial^{3} \eta}{\partial x^{3}} \\
& -\frac{3}{8} c^{*} \eta^{2} \frac{h_{1}^{2}+6 h_{1} h_{2}+h_{2}^{2}}{8\left(h_{1} h_{2}\right)^{2}} * \frac{\partial \eta}{\partial x}+v_{\text {num }} \frac{\partial^{2} \eta}{\partial x^{2}}=0 .
\end{aligned}
$$

The fifth member of Eq. (7) is the second-order component of the nonlinear advection. Figure 5(b) shows that the negative additional nonlinear term slows down and spreads the propagating wave, enabling to retrieve a closer structure to Sim3. In a nutshell, the balance between the first order nonlinear and dispersive terms in the $\mathrm{KdV}$ equation is sufficient to model the general dynamic of high amplitude waves in the pycnocline, while the second order nonlinear term allows to refine the structure of the small scale features of the wave.

Now that it is confirmed that solitary waves are generated at the pycnocline, an important concern is to clarify the influence of the topography shape. In a recent work, Maas ${ }^{24}$ showed by modeling a simple periodic flow over a topography in a linearly stratified fluid, that there exists a large class of ridge shapes for which the generation of internal wave rays is quasi-absent. He proposed a geometrical interpretation of this mechanism, based on the structure of the emitted internal wave rays. This surprising result invites to focus on the role of topography shape on the waves generated in the two homogeneous layers configuration studied in the present article.

\section{TOPOGRAPHIC CONTROL}

\section{A. A topographic criterion for the primary generation}

The objective of this section is to better understand the influence of the topography shape on the primary generation of ISWs. The role of the ridge shape in this process is crucial in various aspects. First, the ridge typical slope controls the amplitude of the interfacial waves $\left(\mathrm{Lamb}^{14}\right)$. In fact, as the ridge slope increases at fixed height, the conversion from horizontal to vertical fluid 

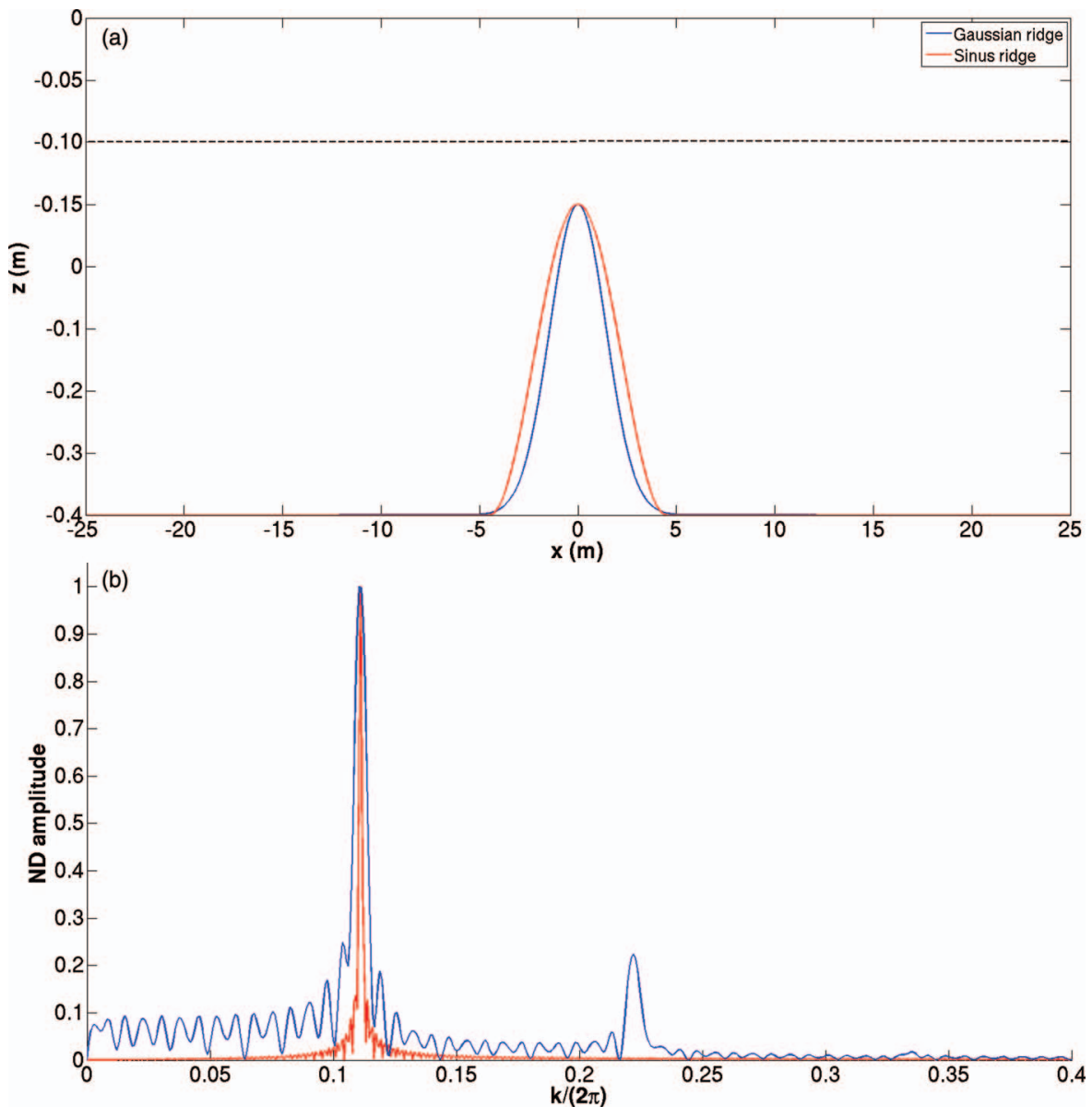

FIG. 6. (a) Profile of the Gaussian (solid line) and sinusoidal (dashed/dotted line) ridges. The position of the pycnocline at rest is recalled by the dashed line. (b) Fourier transform of the periodic Gaussian (dashed line) and sinusoidal (solid line) ridges. Second and third harmonics are visible in the Gaussian ridge spectrum.

displacements becomes larger, leading to interfacial waves of higher amplitude. Moreover, the closer to the interface the ridge top is, the more efficient the vertical interfacial forcing is. These combined effects of the ridge slope and top position are described in the analytical ISW model of Gerkema and Zimmerman 22 via the parameter $\epsilon$. Hence, one can expect that a steep ridge with a top relatively close to the interface potentially generates high amplitude ISWs.

In order to assess the role of the topographic shape, Sim1 is compared with Sim4, for which the ridge shape $h(x)$ is now one period of a sinusoid of wavelength $\lambda_{r}=9 \mathrm{~m}$,

$$
h(x)=\frac{1}{2} h_{0}\left(1+\cos \left(\frac{x}{\lambda_{r}}\right)\right) .
$$

Figure 6(a) displays the shape of the Gaussian and sinusoidal ridges used in Sim1 and Sim4, respectively. Note that the values of $\lambda_{r}$ and $h_{0}$ are chosen, so that the ridges have the same maximal slopes and are equally close to the interface at rest. The stratification and forcing are the same in Sim1 and Sim4. Thus, one could expect that the two ridges generate vertical displacements of similar amplitude in the two experiments. However, the interfacial displacements after 5T, strongly differ as shown in Figure 7. In Sim1, periodic trains of two ISWs with a typical depth of $5 \mathrm{~cm}$ for the first ISW are generated. In Sim4, the interface is locally displaced upwards and downwards due to the ridge oscillation. Away from the ridge, a weak vertical interfacial displacement of order $4 \mathrm{~mm}$ is observed and the associated velocity field is essentially horizontal (not shown). The associated density field at $t=4 T$ is shown in Figure 8. The comparison of these induced interfacial displacements highlights 

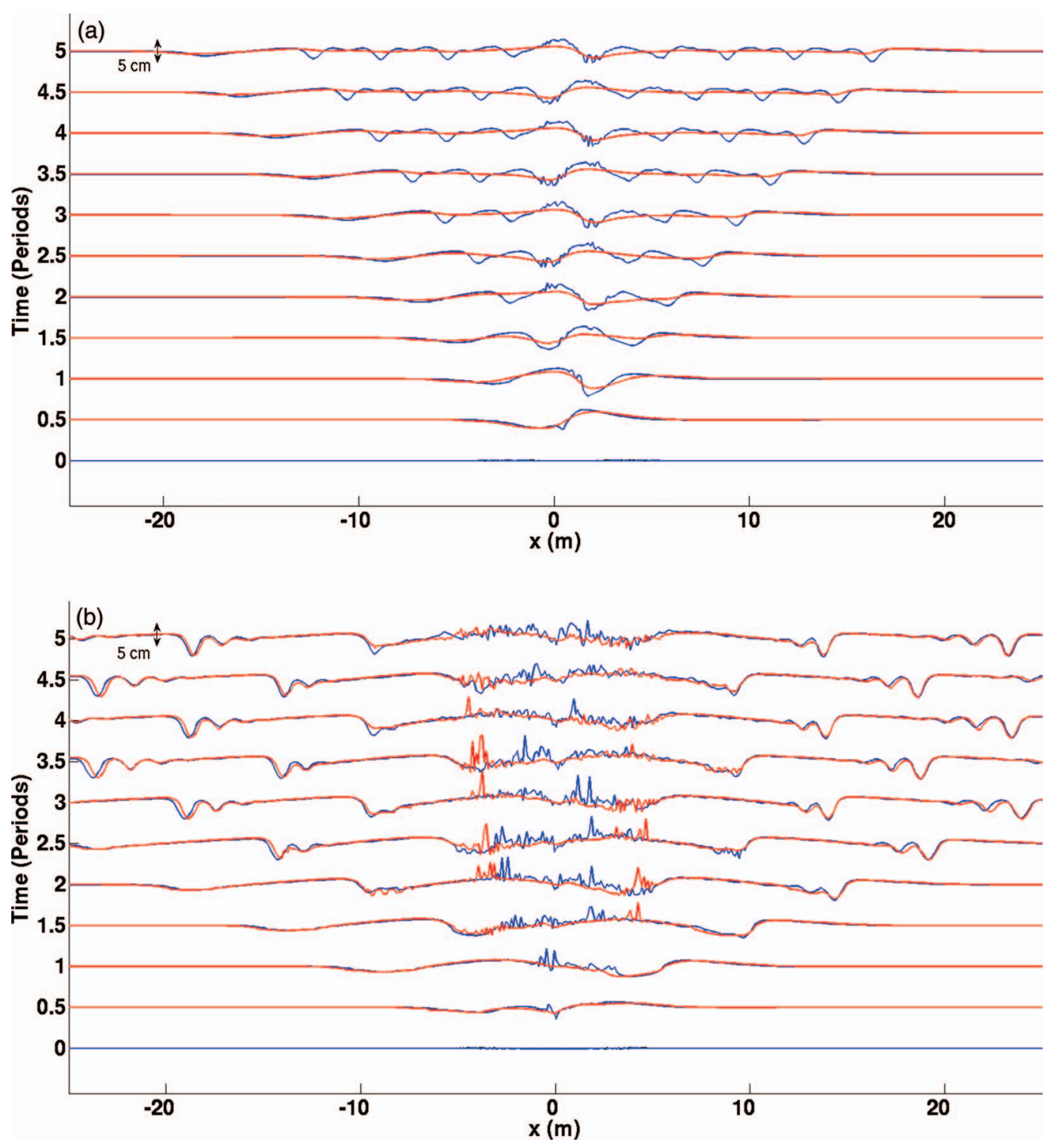

FIG. 7. (a) Mid-pycnocline isopycnal $\left(\rho=1005 \mathrm{~kg} / \mathrm{m}^{3}\right)$ for $t=0 ; 0.5 ; \ldots ; 5 T$, from bottom to top, in Sim1 (dashed, Gaussian ridge, $\epsilon=0.062, \delta=0.47$ ) and in Sim4 (dotted, sinusoidal ridge, $\epsilon=0.062, \delta=0.47$ ). (b) Mid-pycnocline isopycnal $\left(\rho=1018 \mathrm{~kg} / \mathrm{m}^{3}\right)$ for $t=0 ; 0.5 ; \ldots ; 5 T$, from bottom to top, in Sim5 (dashed, Gaussian ridge, $\epsilon=0.062, \delta=0.066$ ), and in Sim6 (dotted, sinusoidal ridge, $\epsilon=0.062, \delta=0.066$ ). The vertical step is $10 \mathrm{~cm}$ between two consecutive time steps.

the crucial role of the topography shape in the primary generation process. In fact, it appears that the amplitude of an interfacial wave with a given wavelength and hence its potential evolution into an ISW is controlled by the topography shape. The latter selects the range of interfacial wavelengths in which energy is efficiently transferred leading to high amplitude, potentially nonlinear, waves or not.

In the context of linear or weakly nonlinear internal waves, it has been demonstrated in various theoretical approaches that the Fourier spectrum of the ridge shape is present in the expression of the energy transfer from the barotropic to the baroclinic tide (Zeilon, ${ }^{25}$ Bell, ${ }^{26}$ Baines, ${ }^{27}$ and Khatiwala $^{28}$ ). Although the interfacial waves are nonlinear in the present case, a Fourier transform of the ridge shapes, shown in Figure 6(b), gives some insights into the wavelength selection by the topography. The Fourier transform are carried out over periodic topographies, consisting in 20 wavelengths $\lambda_{r}$ of the sinusoid given in Eq. (8) in the case of the sinusoidal ridge, and 20 Gaussian 

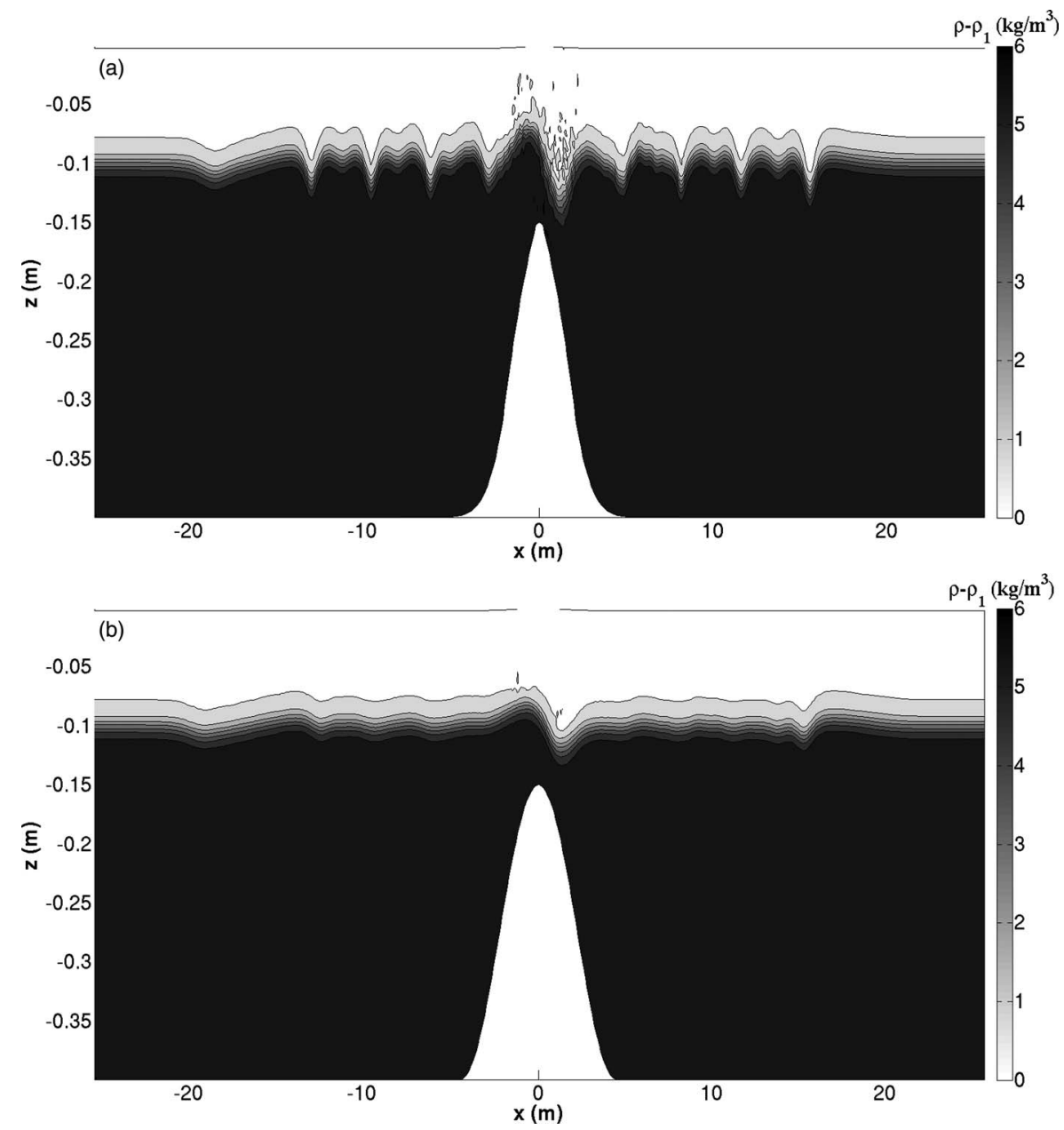

FIG. 8. Symphonie output for the density fields. 8 isopycnal lines at $t=5 T$, in $\operatorname{Sim} 1(\epsilon=0.062, \delta=0.47)$ (a) and in $\operatorname{Sim} 4$ $(\epsilon=0.062, \delta=0.47)(\mathrm{b})$.

ridges spaced by $\lambda_{r}$ in the case of the Gaussian ridge. The mean height of the topography is subtracted before performing the Fourier transform to cancel the continuous component in the spectrum. These modifications allow to discuss the role played by the fundamental and harmonic components present in the topographies. To refer to the periodic ridges, the terms p-Gaussian and p-sinusoidal are used.

The spectrum of the p-sinusoidal ridge is obviously monochromatic. One single peak at $k /(2 \pi)=0.11 \mathrm{~m}^{-1}$ is present corresponding to the wavelength $\lambda_{r}$ of the ridge. The spectrum of the p-Gaussian ridge also exhibits a sharp peak at $k /(2 \pi)=0.11 \mathrm{~m}^{-1}$, while harmonic peaks of smaller amplitude are present (the first and second harmonics are visible in Figure 6(b)).

Figures 6(a) and 6(b) permit an interpretation of the topographic control exerted by the ridge. The oscillation of the ridge provokes a local distortion of the interface above the ridge whose shape highly depends on the topography: a sharp (respectively, smooth) slope would induce a steep (respectively, weak) interfacial displacement. Besides, the topographic control increases with the height of the ridge: the closer to the pycnocline, the larger the impact on the interfacial distortion. To provide a clear picture, we assume that the ridge is infinitely close to the pycnocline. In the case of the sinusoidal ridge, the local distortion takes the shape of one wavelength of a sinusoid $\left(\lambda_{r}\right)$. If the interfacial wavelength $\lambda$ differs from $\lambda_{r}$, the local, monochromatic, distortion cannot propagate efficiently as an interfacial wave and is confined above the ridge as seen in Figure 7(a). On the contrary, we expect that for an interfacial wavelength $\lambda$ matching $\lambda_{r}$, the local distortion evolves into propagating interfacial waves of high amplitude, which disintegrates into ISWs. 
To assess this assumption, two simulations are performed, Sim5 with the same Gaussian ridge as in Sim1 and Sim3, Sim6 with the same sinusoidal ridge as in Sim4. The only change is the density jump $\Delta \rho=36 \mathrm{~kg} / \mathrm{m}^{3}$ adjusted so that $\lambda=9.75 \mathrm{~m} \approx \lambda_{r}$. In the course of Sim6, the behavior of the interfacial displacement, shown in Figure 7(b), is strikingly different from Sim4: trains of relatively deep ISWs develop, which supports the idea of the topographic control in the primary generation process. In fact, the interfacial wavelength in Sim6 matches the optimal wavelength imposed by the sinusoidal ridge: important vertical displacements propagating in the pycnocline evolve into ISWs due to the balance between nonlinear and dispersive effects. In addition, the behavior of the ISWs trains is almost identical, in terms of shape and amplitude, to Sim5, since the nonlinear and nonhydrostatic coefficients, controlling the ISW structure, are the same in the two experiments. In addition to the balance between nonlinear and non-hydrostatic effects, the ridge shape must be taken into account to forecast the primary generation of ISW, as shown above. High amplitude ISWs can only be generated providing the interfacial wavelength falls within the range of wavelengths at which the ridge causes substantial vertical displacements. For the sinusoidal ridge, this condition can be simply expressed with the ratio

$$
\lambda_{n d}=\frac{\lambda}{\lambda_{r}} \approx 1,
$$

where $\lambda_{n d}$ is the nondimensional wavelength. Equation (9) appears to be a spatial selection criterion for an efficient primary generation of ISWs. This criterion is analogous to the one proposed by Akylas et al. ${ }^{11}$ for the secondary generation process. In the latter case, the matching of the horizontal wavelength of the forcing internal wave ray $\lambda_{I W R}$ on one hand and the interfacial wavelength $\lambda$ on the other hand leads to an efficient secondary generation of ISWs. Hence, the parameter $\alpha$ introduced by Akylas et al. ${ }^{11}$ and proportional to $\lambda_{I W R} / \lambda$ is the counterpart of $\lambda_{n d}$ for the secondary generation process. The condition $\alpha=1$ can also be expressed as a matching between phase speeds, as the forcing is a propagating motion (Grisouard et al. ${ }^{12}$ ).

In the case of $\operatorname{Sim} 4, \lambda_{n d} \approx 0.4$, weak vertical displacements are initiated in the pycnocline at this wavelength and the velocity field is essentially horizontal (not shown). For Sim6, $\lambda_{n d} \approx 1.1$ and ISWs are efficiently generated. We propose the term topographic control to refer to this control exerted by the topography shape on the primary generation of ISWs.

In Sim4-Sim6, as well as in oceanic configurations, the topography is at a finite distance from the pycnocline, the local interfacial distortion does not exactly match the ridge shape. Hence the selection criterion is potentially looser than in the previous description: for a realistic topography, the wavelength range for which the energy transfers from the ridge to the interfacial wave are substantial not only depends on the ridge shape but also on the ridge-pycnocline distance. To summarize, the spatial selection for the primary generation is expected to be stronger in regions where the topography is relatively close to the interface, and forces quasi-monochromatic interfacial displacements. It is therefore interesting to check how the structure of ISWs generated over nonmonochromatic ridges (i.e., over ridges forcing isopycnal displacements at various wavelengths) is affected by the topography shape.

\section{B. Extension to a non-monochromatic ridge}

The topographic control appears less selective for a Gaussian ridge in so far as ISWs are generated in both simulations Sim1 and Sim5. In fact, the presence of harmonics in the spectrum of the periodic Gaussian ridge permits to provide substantial energy to propagating interfacial wave at shorter wavelengths.

In order to show the effects of the topographic control for a non-monochromatic ridge, two other series of simulations, Sim7 and Sim8, using different Gaussian ridges are performed. The e-folding and bottom widths are $l=1 \mathrm{~m}$ and $\lambda_{r}=4.5 \mathrm{~m}$ in Sim7 (respectively, $l=2 \mathrm{~m}$ and $\lambda_{r}=9 \mathrm{~m}$ in Sim8). The forcing amplitude is $A=10 \mathrm{~cm}$ in $\operatorname{Sim} 7$ and $A=20 \mathrm{~cm}$ in $\operatorname{Sim8}$, so $\epsilon$ has the same value in both cases. For each series, the interfacial wavelength is varied through the density jump in the pycnocline. After 5T, the depth of the first ISW of the second train emitted to the left of the 


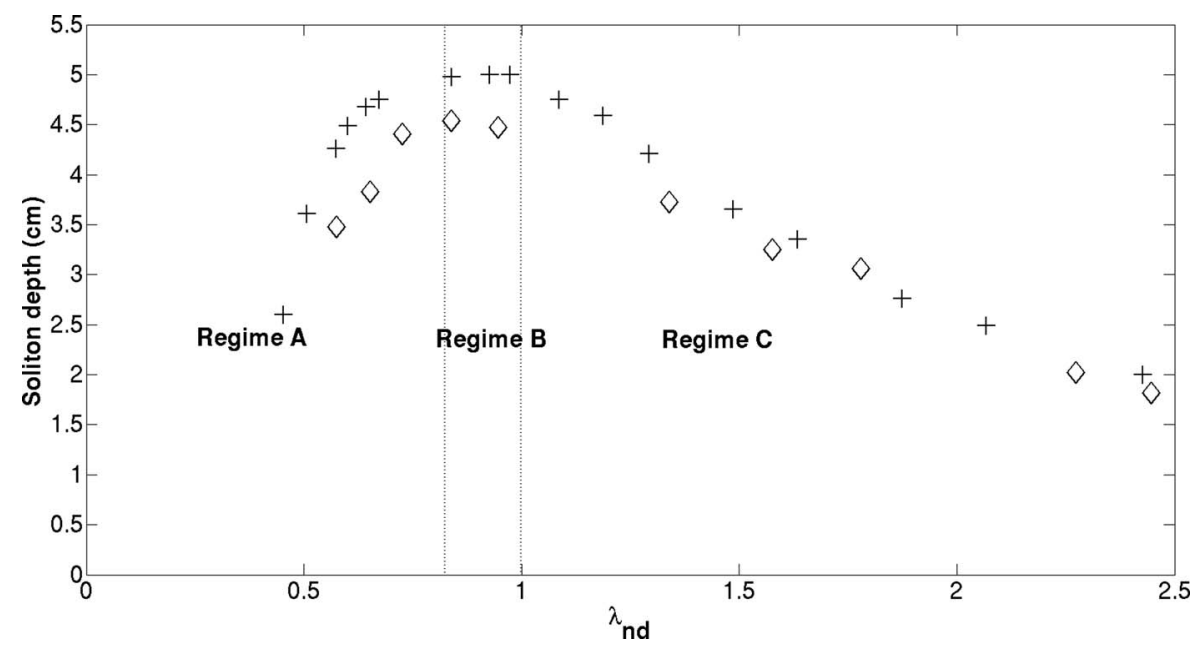

FIG. 9. Evolution of the first ISW depth with respect to $\lambda_{n d}$ for $\operatorname{Sim} 7\left(\lambda_{r}=4.5 \mathrm{~m}, \epsilon=0.062, \delta=\right.$ [0.068: 1.192], diamond) and for $\operatorname{Sim8}\left(\lambda_{r}=9 \mathrm{~m}, \epsilon=0.062, \delta=[0.015: 1.192],+\right)$. The uncertainty on the depth measurement is $\pm 2 \mathrm{~mm}$. The maximum depth is reached for the same range of $\lambda_{n d}$, showing that the ridge width controls the maximum energy input into ISWs.

ridge (shown in gray, for example, in Figure 4) is measured in all experiments. The evolution of the first ISW depth against $\lambda_{n d}$ is plotted in Figure 9.

Overall, the two series exhibit similar shapes for varying wavelength, with a slightly lower amplitude depth for Sim7 than for Sim8. Three different behaviors are observed in the evolution of the depth with the forcing wavelength. In regime A $\left(0.5 \leq \lambda_{n d} \leq 0.8\right)$, the ISW depth rapidly increases to reach a plateau of magnitude $5 \mathrm{~cm}$, starting at $\lambda_{n d} \approx 0.8$. The depth of the first soliton is here subject to the topographic control: according to the Fourier spectrum of the periodic Gaussian ridge shown in Figure 6(b), the contribution of low order harmonics rises for increasing interfacial wavelength. Consequently, ISWs are relatively weak for the short wavelengths, and they progressively deepen as the energy transfer to propagating interfacial waves becomes more efficient for increasing wavelength. Notice that dispersion is sufficiently strong to sustain trains of two ISWs even for short interfacial wavelengths. In regime B $\left(0.8 \leq \lambda_{n d} \leq 1\right)$, strong ISWs are generated. In this range of interfacial wavelengths, both the fundamental and the first harmonics of the ridge spectrum contribute to the energy transfer to interfacial waves, thereby permitting the generation of high amplitude ISWs. The maxima are reached for the same range of $\lambda_{n d}$ in the two series, highlighting the topographic control for a Gaussian ridge: the ridge width imposes the bandwidth at which most energy is transferred to interfacial waves. In regime C, $\left(\lambda_{n d} \geq 1\right)$, the first ISW depth progressively decreases almost linearly. For increasing long wavelengths, the number of ISWs in a train increases as dispersive effects become too weak to sustain nonlinearity. Thus, the energy splitting within an ISW train causes the amplitude weakening of the first ISW of the train. Contrary to regimes $\mathrm{A}$ and $\mathrm{B}$, the nonlinearity/dispersion balance also has a strong influence on the ISW depth, along with topographic control.

In order to detail the evolution of the shape of an ISWs train, the depth of each ISW in a train is measured in Sim8. Figure 10 displays the evolution of the $n$th ISW depth (with $n=1$; $2 ; 3 ; 4$, the ISWs being ranked by their position in the train) for varying $\lambda_{n d}$. For short enough $\lambda_{n d}$, only two solitons are present in the train, both showing a bell-shaped evolution with a sharp increase at short wavelengths due to the topographic control. For increasing $\lambda_{n d}$, the third and fourth solitons appear owing to the progressive weakening of dispersive effects. Solitons are always ranked by decreasing amplitude as their propagation speed increases with depth. Note that the optimal wavelength regarding the maximum soliton depth is reached for a higher wavelength as the soliton rank increases. In fact, for $0.45 \leq \lambda_{n d} \leq 0.8$, the nonlinear/dispersive balance leads to stable train of two solitons. As $\lambda_{n d}$ increases, non-hydrostatic dispersion weakens, enabling the existence of a third soliton in the train. The growth of the third soliton occurs at the expense of the solitons of ranks 1 


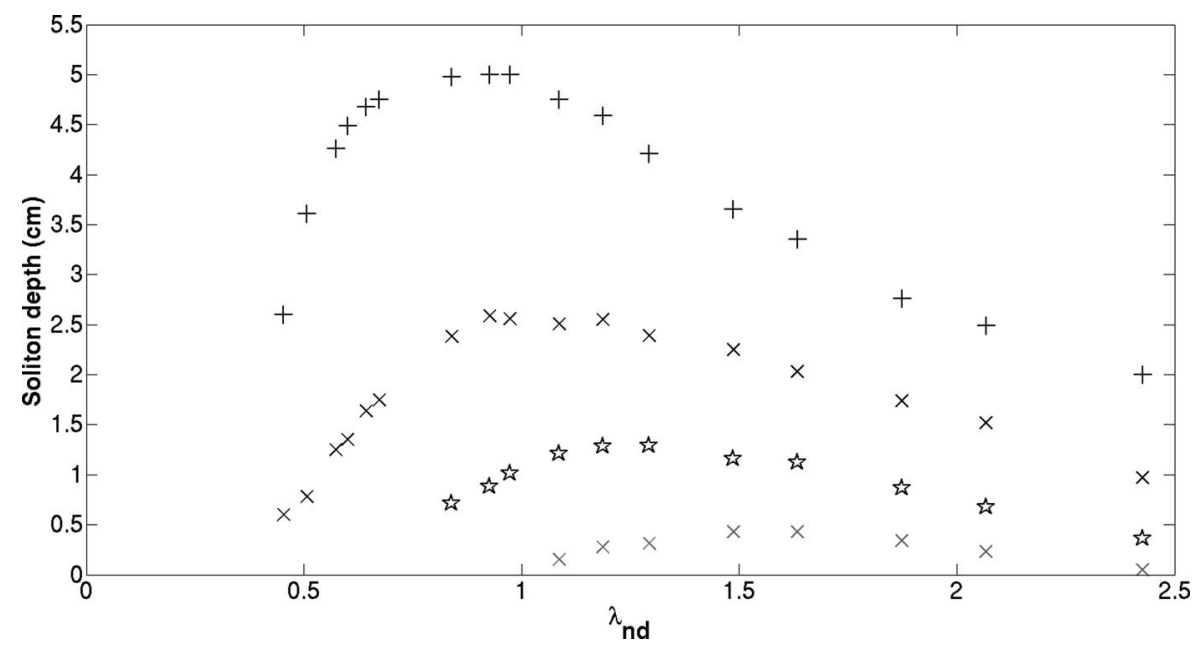

FIG. 10. Black $+($ respectively, $\times$, star, gray $\times)$ : Evolution of the first (respectively, second, third, fourth) soliton depth with respect to $\lambda_{n d}$ for $\operatorname{Sim8}\left(\lambda_{r}=9 \mathrm{~m}, \epsilon=0.062, \delta=[0.015: 1.192]\right)$. The uncertainty on the depth measurement is $\pm 2 \mathrm{~mm}$. For increasing $\lambda_{n d} \geq 1$, the number of ISWs in a train increases due to the weakening of non-hydrostatic effects, while the depth of a given ISW decreases. The ISW depth reaches a topographically controlled maximum for at least the first three ISWs.

and 2, whose amplitude decreases. For $\lambda_{n d} \geq 1$, the interfacial wave disintegrates into four solitons, and the solitons 1, 2, 3 start leaking energy to the fourth soliton. Hence, a soliton of fixed rank will reach a maximum amplitude, for increasing wavelength, before weakening owing to the birth of the next order soliton, which will in turn follow a similar evolution.

To sum up, the comparison of interfacial waves generated over a monochromatic (sinusoidal) ridge, and a non-monochromatic (Gaussian) ridge with a more complex wavelength spectrum permits to better understand the selection criterion for the primary generation of ISWs. This selection criterion is spatial: the interfacial wavelength must be close enough to the intrinsic wavelength of the ridge to allow interfacial wave propagation. Provided the wave amplitude is large enough, nonlinear and non-hydrostatic effects can balance to generate ISWs. The Gaussian ridge, which displays a broader wavelength spectrum, also controls the generation of ISWs, since the maximum ISW depth occurs at a wavelength close to the ridge width. In an oceanic context, the study of the topographic features at the ocean bottom may help to forecast better the hotspots for the primary generation of ISWs.

\section{CONCLUSION}

Direct numerical simulations using Symphonie-NH have been investigated to better understand the primary generation of ISWs at a pycnocline above a ridge of given shape. The generation process of high amplitude interfacial waves relying on the nonlinear/dispersion balance has been described. Then the numerical outputs of the pycnocline displacement in Symphonie-NH have been compared to the numerical outputs of a KdV scheme. The comparison showed that the nonlinear and dispersive terms in the simple KdV scheme satisfactorily describe the wave dynamics issued from the numerical model. It showed also that an extended KdV scheme enables to retrieve, with a good accuracy, smaller scale features in the ISWs, enabling to prove that the propagating interfacial waves were indeed ISWs.

The role of the topography shape has then been investigated by varying the ridge shape. The use of a sinusoidal ridge has enabled to show the spatial resonance imposed by the topography, with respect to the generation and propagation of ISWs. Two series of simulations with Gaussian ridges of different base width, confirmed the topographic control assumption in the primary generation of ISWs. With the hindsight of the present study, it can be inferred from the condition given by Eq. (9) that an important condition for ISWs primary generation is the accordance between the wavelength favored by the ridge on one side, and the interfacial wavelength imposed by the stratification above the 
ridge and the forcing period on the other side. The ratio of these two quantities, issued from realistic topographies and stratification, may allow to localize high amplitude ISWs primary generation zones in geophysical flows, using a Fourier transform method to obtain the bandwidth at which spatial resonance may occur. In order to get closer to realistic configurations, the role played by the velocity shear in the topographic selection criterion, should also be investigated.

Secondarily generated ISWs could also be subject to a topographic control, in an indirect way. As said above, the interaction between the internal wave ray and the pycnocline may generate ISWs, providing a spatial resonance between the horizontal wavelength of the internal wave ray and the interfacial wavelength occurs (Akylas et al. ${ }^{11}$ ). The topography plays a role in the efficiency the secondary generation process, by controlling the structure of the internal wave ray for a given fixed stratification (Dossmann et al. ${ }^{29}$ and references therein). Recent numerical simulations performed by Grisouard et al. ${ }^{12}$ gave an interesting description on the secondary generation process for a pycnocline of finite thickness. They extracted a selection criterion relying on a horizontal phase speed matching between the internal wave beam and a normal mode with an important interfacial signature. Future studies will use direct numerical simulations to complete the description of the secondary generation process with a particular focus on the topography shape. Along with the present work, it will contribute to improve our understanding of topographically generated ISWs at a pycnocline and to give insights for the development of ISWs forecasting tools in the geophysical context.

\section{ACKNOWLEDGMENTS}

This work has been supported by LEFE-IDAO Programme "ondes et marées internes dans l'océan" (LEFE-IDAO-07/2) and ANR "PIWO" Contract No. ANR-08-BLAN-0113. Y. Dossmann's $\mathrm{Ph} . \mathrm{D}$. dissertation is funded by a MNERT scholarship. We thank the POC team (LA, UMR 5560, Paul Sabatier University and CNRS and LEGOS, UMR 5566, CNES, CNRS, IRD Paul Sabatier University) for their kind support. Numerical experiments were performed on the French supercomputer center HYPERION, through projects p1052 and p1054 and on the Laboratoire d'Arologie cluster (a great thanks to the LA computing team).

\section{APPENDIX: NUMERICAL KdV SCHEME}

The discretization of the KdV equation (6), using a centered scheme for spatial discretization, (spatial step $\Delta x=5 \mathrm{~mm}$ ), and a time-advancing scheme for temporal discretization (time step $\left.\Delta t=6 e^{-6} \mathrm{~s}\right)$ is given by the following equations:

$$
\begin{array}{r}
\eta(i x, i t)=\eta(i x, i t-1)+\left[\operatorname{lprop}(i x, i t-1)+\operatorname{nlprop}_{1}(i x, i t-1)+\right. \\
\left.\operatorname{nlprop}_{2}(i x, i t-1)+\operatorname{disp}(i x, i t-1)+\operatorname{visc}(i x, i t-1)\right] \times \Delta t
\end{array}
$$

with

$$
\operatorname{lprop}(i x, i t-1)=-c^{*} \times \frac{\eta(i x+1, i t-1)-\eta(i x-1, i t-1)}{2 \Delta x},
$$

$$
\begin{aligned}
\operatorname{nlprop}_{1}(i x, i t-1) & = \\
& -3 / 2 \frac{h_{1}-h_{2}}{h_{1} h_{2}} c^{*} \times \eta(i x, i t-1) \times \frac{\eta(i x+1, i t-1)-\eta(i x-1, i t-1)}{2 \Delta x}, \\
\operatorname{nlprop}_{2}(i x, i t-1) & =1 / 8 \frac{h_{1}^{2}+6 h_{1} h_{2}+h_{2}^{2}}{\left(h_{1} h_{2}\right)^{2}} c^{*} \times \frac{\eta(i x+1, i t-1)^{3}-\eta(i x-1, i t-1)^{3}}{2 \Delta x},
\end{aligned}
$$




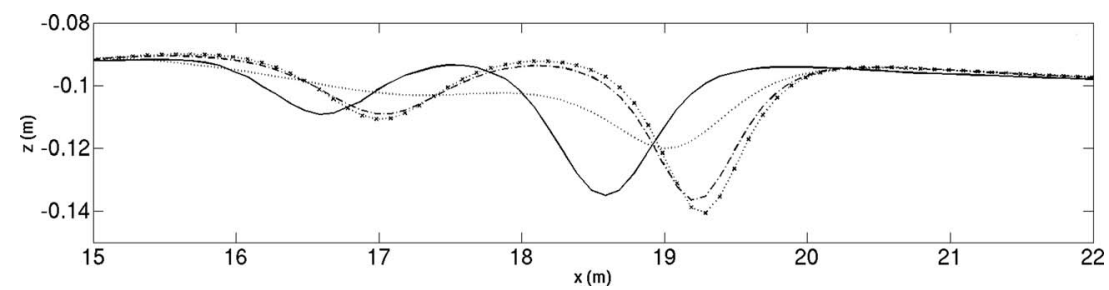

FIG. 11. (Solid line) Mid-pycnocline isopycnal $\left(\rho=1005 \mathrm{~kg} / \mathrm{m}^{3}\right)$ in $\mathbf{S i m 3}$, zoomed on one wavelength to the right of the ridge, at $t=4 T$. Output of the simple KdV scheme, with $v_{\text {num }}=10^{-6} \mathrm{~m}^{2} / \mathrm{s}$ (dotted $/ \mathrm{plus}$ ), $v_{\text {num }}=10^{-4} \mathrm{~m}^{2} / \mathrm{s}$ (dashed), $v_{\text {num }}$ $=10^{-3} \mathrm{~m}^{2} / \mathrm{s}$ (dotted), at $t=4 T$. The isopycnal displacement in Sim3 is close to the simple KdV scheme output with $v_{\text {num }}$ $=10^{-4} \mathrm{~m}^{2} / \mathrm{s}$.

$$
\begin{aligned}
& \operatorname{disp}(i x, i t-1)= \\
& -1 / 6 h_{1} h_{2} c^{*} \times \frac{\eta(i x+3, i t-1)-3 \eta(i x+1, i t-1)+3 \eta(i x-1, i t-1)-\eta(i x-3, i t-1)}{8 \Delta x^{3}}, \\
& \operatorname{visc}(i x, i t-1)=v_{\text {num }} \times \frac{\eta(i x+1, i t-1)-2 \eta(i x, i t-1)+\eta(i x-1, i t-1)}{4 \Delta x^{2}} .
\end{aligned}
$$

The linear longwave interfacial speed $c^{*} \approx 0.08 \mathrm{~m} / \mathrm{s}$ is calculated from Eq. (3), with $h_{1}=10 \mathrm{~cm}, h_{2}$ $=30 \mathrm{~cm}$, and $\Delta \rho=10 \mathrm{~kg} / \mathrm{m}^{3}$. The terms $\operatorname{lprop}(i x, i t-1), \operatorname{nlprop}_{1}(i x$, it -1$), \operatorname{nlprop}_{2}(i x$, it -1$)$ are the discretized expressions of the linear propagation, and of the first and second-oder nonlinear advection terms, respectively, calculated at the grid point $i x$, and at the time step it -1 . nlprop $_{2}(i x, i t-1)$ is the second-order nonlinear advection term, only used in the extended $\mathrm{KdV}$ scheme (6), and set to zero in the simple $\mathrm{KdV}$ scheme. $\operatorname{disp}(i x, i t-1)$ is the discretized expression of the dispersive term. visc $(i x, i t-1)$ is a parameterized viscous term used to stabilize the $\mathrm{KdV}$ scheme. The value $v_{\text {num }}=10^{-4} \mathrm{~m}^{2} / \mathrm{s}$ for the numerical viscosity permits to limit the increase of the wave amplitude due to numerical discretization, while preserving the physical features of the wave, as shown in Figure 11. Periodic boundary conditions are used.

${ }^{1}$ T. Gerkema and J. T. F. Zimmerman, An Introduction to Internal Waves, Lecture Notes (Royal NIOZ, 2008).

${ }^{2}$ R. H. J. Grimshaw, L. A. Ostrovsky, V. I. Shrira, and Y. A. Stepanyants, "Long nonlinear surface and internal gravity waves in a rotating ocean," Surv. Geophys. 19, 289-338 (1998).

${ }^{3}$ T. Gerkema, "Internal and interfacial tides: Beam scattering and local generation of solitary waves," J. Mar. Res. 59, 227-255 (2001).

${ }^{4}$ L. S. Kantha and C. A. Clayson, "An improved mixed layer model for geophysical applications," J. Geophys. Res. 99, 25235-25266, doi:10.1029/94JC02257 (1994).

${ }^{5}$ P. Hyder, D. R. G. Jeans, E. Cauquil, and R. Nerzic, "Observations and predictability of internal solitons in the northern Andaman Sea,” Appl. Ocean. Res. 27, 1-11 (2005).

${ }^{6}$ Z. Lai, C. Chen, R. C. Beardsley, B. Rothschild, and R. Tian, "Impact of high-frequency nonlinear internal waves on plankton dynamics in Massachusetts Bay," J. Mar. Res. 68, 259-281 (2010).

${ }^{7}$ J. Apel, J. Holbrook, A. Liu, and J. Tsai, "The Sulu Sea internal soliton experiment," J. Phys. Oceanogr. 15, 1625-1651 (1985).

${ }^{8}$ R. D. Pingree and G. T. Mardell, "Solitary internal waves in the Celtic Sea," Prog. Oceanogr. 14, 431-441 (1985).

${ }^{9}$ A. L. New and R. D. Pingree, "Large-amplitude internal soliton packets in the central Bay of Biscay," Deep-Sea Res., Part I 37, 513-524 (1990).

${ }^{10}$ A. L. New and R. D. Pingree, "Local generation of internal soliton packets in the central Bay of Biscay," Deep-Sea Res., Part I 39, 1521-1534 (1992).

${ }^{11}$ T. R. Akylas, R. H. J. Grimshaw, S. R. Clarke, and A. Tabaei, "Reflecting tidal wave beams and local generation of solitary waves in the ocean thermocline," J. Fluid Mech. 593, 297 (2007).

${ }^{12}$ N. Grisouard, C. Staquet, T. Gerkema et al., "Generation of internal solitary waves in a pycnocline by an internal wave beam: A numerical study," J. Fluid Mech. 676, 491 (2011).

${ }^{13}$ T. Gerkema, "Nonlinear dispersive internal tides: Generation models for a rotating ocean," Ph.D. dissertation (Universiteit Utrecht, 1994).

${ }^{14}$ K. G. Lamb, "Numerical experiments of internal wave generation by strong tidal flow across a finite amplitude bank edge," J. Geophys. Res. 99, 843-864, doi:10.1029/93JC02514 (1994).

${ }^{15}$ F. Auclair, C. Estournel, J. W. Floor, M. Herrmann, C. Nguyen, and P. Marsaleix, "A non-hydrostatic algorithm for free-surface ocean modelling," Ocean Model. 36, 49-70 (2011). 
${ }^{16}$ A. Warn-Varnas, J. Hawkins, K. Lamb, S. Piacsek, S. A. Chin-Bing, D. King, and G. Burgos, "Solitary wave generation dynamics at Luzon Strait," Ocean Model. 31, 9-27 (2010).

${ }^{17}$ A. L. New and R. Pingree, "An intercomparison of internal solitary waves in the bay of biscay and resulting from Korteweg-de Vries-type theory," Prog. Oceanogr. 45, 1-38 (2000).

${ }^{18}$ A. F. Blumberg and G. L. Mellor, "A description of a three-dimensional coastal circulation model," Coastal Estuarine Sci. 4, 1-16 (1987).

${ }^{19}$ J. W. Floor, “Analyse énergétique des marées internes: de la génération au mélange induit," Ph.D. dissertation (Université Paul Sabatier Toulouse III, 2009).

${ }^{20}$ J. Floor, F. Auclair, and P. Marsaleix, "Energy transfers in internal tide generation, propagation and dissipation in the deep ocean," Ocean Model. 38, 22-40 (2011).

${ }^{21}$ C. Knigge, D. Etling, A. Paci, and O. Eiff, "Laboratory experiments on mountain-induced rotors," Q. J. R. Meteorol. Soc. 136, 442-450 (2010).

${ }^{22}$ T. Gerkema and J. Zimmerman, "Generation of nonlinear internal tides and solitary waves," J. Phys. Oceanogr. 25, 1081-1094 (1995).

${ }^{23}$ T. Dauxois and M. Peyrard, Physics of Solitons (Cambridge University Press, New York, 2006).

${ }^{24}$ L. R. M. Maas, “Topographies lacking tidal conversion,” J. Fluid Mech. 684, 5-24 (2011).

${ }^{25}$ N. Zeilon, On Tidal Boundary-Waves and Related Hydrodynamical Problems (Kongl. Svenska Vetenskaps Akademiens Handlingar, Stockholm, 1912), Vol. 47.

${ }^{26}$ T. H. Bell, "Lee waves in stratified flows with simple harmonic time-dependance," J. Fluid Mech. 67, 705-722 (1975).

${ }^{27}$ P. G. Baines, "On internal tide generation models," Deep-Sea Res., Part I 29, 307-338 (1982).

${ }^{28}$ S. Khatiwala, "Generation of internal tides in an ocean of finite depth: Analytical and numerical calculations," Deep-Sea Res., Part I 50, 3-21 (2003).

${ }^{29}$ Y. Dossmann, A. Paci, F. Auclair, and J. W. Floor, "Simultaneous velocity and density measurements for an energy-based approach to internal waves generated over a ridge,” Exp. Fluids 51, 1013-1028 (2011). 